\title{
ISOMETRIES BETWEEN FUNCTION SPACES
}

\author{
KRZYSZTOF JAROSZ AND VIJAY D. PATHAK
}

\begin{abstract}
Surjective isometries between some classical function spaces are investigated. We give a simple technical scheme which verifies whether any such isometry is given by a homeomorphism between corresponding Hausdorff compact spaces. In particular the answer is positive for the $C^{1}(X), \mathrm{AC}[0,1]$, $\operatorname{Lip}_{\alpha}(X)$ and $\operatorname{lip}_{\alpha}(X)$ spaces provided with various natural norms.
\end{abstract}

1. Introduction. Let $A$ and $B$ be Banach spaces. By an isometry from $A$ onto $B$ we mean a linear, norm preserving and surjective map between these Banach spaces. The isometries of most of the well-known Banach spaces have been described. The classical Banach-Stone theorem states that any isometry from $C(X)$ onto $C(Y)$ is induced by a homeomorphism of $Y$ and $X$. This result has been extended to various other Banach spaces by Nagasawa for function algebras [16]; by Amir [1], Cambern [3] and Cengiz [7] for regular subspaces; by Cambern and Pathak [5], Pathak [17] and Pathak and Vasavada [19] for spaces of differentiable functions; by de Leeuw [15] and Roy [21] for Lipschitz functions; by Pathak [18], Vasavada [22] and Rao and Roy [20] for absolutely continuous functions and by many other authors. In all the above-mentioned papers the following situation was considered.

Let $A$ be a subspace of a Banach space $C(X)$, which separates points of $X$, and let $T_{A}$ be a linear map from $A$ into a Banach space $V$. We assume the complete norm on $A$ is given by one of the following formulas:

$$
\|f\|=\max \left(\|f\|_{\infty},\left\|T_{A} f\right\|\right) \quad \text { for } f \in A,
$$

where by $\|\cdot\|_{\infty}$ we denote the usual sup-norm on $C(X)$;

$$
\begin{gathered}
\|f\|=\|f\|_{\infty}+\left\|T_{A} f\right\| \quad \text { for } f \in A \\
\|f\|=\sup \left\{|f(x)|+\left|T_{A} f(x)\right|: x \in X\right\} \quad \text { for } f \in A,
\end{gathered}
$$

where, in this case, we assume that $V=C(X)$.

For example the space $C^{1}(X), X \subset R$, is defined by a map $T: C^{1}(X) \rightarrow$ $C(X): T f=f^{\prime}$, via the formula $(\mathrm{M}),(\Sigma)$ or $(\mathrm{C})$. The space $\mathrm{AC}[0,1]$ of absolutely continuous functions is defined by a map $T: \mathrm{AC}[0,1] \rightarrow L^{1}[0,1]: T f=f^{\prime}$. The space $\operatorname{Lip}_{\alpha}(X), X$-metric space, $0<\alpha \leq 1$, is defined by

$$
T: \operatorname{Lip}_{\alpha}(X) \rightarrow C(\beta(X \times X \backslash\{(x, x): x \in X\})): T f(x, y)=\frac{f(x)-f(y)}{(d(x, y))^{\alpha}} .
$$

Assume next that $B$ is a subspace of $C(Y)$, which separates points of $Y$, and that the norm on $B$ is given by a map $T_{B}: B \rightarrow U$, via the same formula as the

Received by the editors June 13, 1986.

1980 Mathematics Subject Classification (1985 Revision). Primary 46E15, 46J10. 
norm of $A$. The question arises whether any isometry $\Phi$ from $A$ onto $B$ is of the canonical form

$$
\Phi(f)(y)=\chi(y) \cdot f \circ \varphi(y), \quad f \in A, y \in Y,
$$

where $\varphi$ is a homeomorphism from $Y$ onto $X$ and $\chi$ is a scalar valued function defined on $Y$ such that $|\chi| \equiv 1$.

In this note we give a very simple, elementary scheme to verify the abovementioned problems. This scheme covers all the results we mentioned at the beginning of the introduction. The results hold in both real and complex cases.

2. Definitions and notation. In this note we use the standard Banach space terminology. We denote by ext $V$ the set of all extreme points of the closed unit ball $V_{1}$ of the space $V$; by $V^{*}$ we denote the space of all continuous linear forms on $V$. If $A$ is a subspace of $C(X)$ then we identify a point $x$ of $X$ with a linear functional $\delta_{x}$ on $A$ defined by $A \ni f \mapsto f(x)$. By $S$ we denote the set of all scalars of modulus one. In this paper, if we do not specify the set of scalars, we mean that the result holds both in the real and in the complex case.

Let $\tilde{A}$ be a normed subspace of a Banach space $C(W)$, both with the usual supnorm. By a standard result $[8$, vol. 1, p. 441$]$ every extreme point of $\tilde{A}_{1}^{*}$ is of the form $\lambda \delta_{w}$, where $w \in W$ and $|\lambda|=1$.

Note that if the norm on $A$ is defined by the formula (M) then there is an isometric embedding $A \ni f \mapsto \tilde{f} \in \tilde{A} \subset C(W)$, where $W=X \cup V_{1}^{*}$, defined by

$$
\begin{aligned}
\tilde{f}(x) & =f(x), & & x \in X, \\
\tilde{f}\left(v^{*}\right) & =v^{*}\left(T_{A} f\right), & & v^{*} \in V_{1}^{*} .
\end{aligned}
$$

Hence any extreme point of $A_{1}^{*}$ is of the form

$$
f \mapsto \alpha f(x), \quad \text { where } x \in X \text { and } \alpha \in S,
$$

or of the form

$$
f \mapsto F \circ T_{A}(f), \quad \text { where } F \in \operatorname{ext} V^{*} .
$$

If the norm on $A$ is defined by $(\Sigma)$ then a map $A \ni f \mapsto \tilde{f} \in \tilde{A} \subset C\left(X \times V_{1}^{*}\right)$ defined by

$$
\tilde{f}\left(x, v^{*}\right)=f(x)+v^{*}\left(T_{A} f\right), \quad\left(x, v^{*}\right) \in X \times V_{1}^{*}
$$

is an isometry. Hence any extreme point of $A_{1}^{*}$ is of the form

$$
f \mapsto \alpha f(x)+F \circ T_{A}(f), \quad \text { where } x \in X, F \in \operatorname{ext} V, \alpha \in S \text {. }
$$

If the norm on $A$ is defined by (C) then a suitable choice is $W=X \times S$ and

$$
\tilde{f}(x, \lambda)=f(x)+\lambda\left(T_{A} f\right)(x), \quad x \in X, \lambda \in S .
$$

Hence any extreme point of $A_{1}^{*}$ is given by

$$
f \mapsto \alpha f(x)+\beta T_{A} f(x)
$$

where $x \in X, \alpha, \beta \in S$.

Let $A$ be a subspace of a space $C(X), X$ compact Hausdorff space. We say that $A$ is an $M$-subspace of $X, \Sigma$-subspace of $X$ or $C$-subspace of $X$ if there is a Banach space $V$ and a linear map $T_{A}: A \rightarrow V$ such that

(1) the norm on $A$ is given by the formula $(\mathrm{M}),(\Sigma)$ or $(\mathrm{C})$, respectively, 
(2) for any $x_{1}, x_{2} \in X$, functionals $\delta_{x_{1}}$ and $\delta_{x_{2}}$ are linearly independent, and if the corresponding assumptions listed below are satisfied:

( $\left.3_{\mathrm{M}}\right) X_{0}=\left\{x \in X: \delta_{x} \in \operatorname{ext} A^{*}\right\}$ is a dense subset of $X$,

$\left(3_{\Sigma}\right)$ there is an $F_{0}$ in ext $V^{*}$ such that

$$
X_{0}=\left\{x \in X ; \forall \alpha \in S, \delta_{x}+\alpha F_{0} \circ T_{A} \in \operatorname{ext} A^{*}\right\}
$$

is a dense subset of $X$,

$\left(3_{\mathrm{C}}\right) X_{0}=\left\{x \in X: \forall \alpha, \beta \in S, \alpha \delta_{x}+\beta \delta_{x} \circ T_{A} \in \operatorname{ext} A^{*}\right\}$ is a dense subset of $X$;

$\left(4_{\Sigma}\right)$ if $\alpha \delta_{x}+F \circ T_{A}=\alpha^{\prime} \delta_{x^{\prime}}+\beta^{\prime} F^{\prime} \circ T_{A}$, where $F, F^{\prime} \in \operatorname{ext} V^{*}, x, x^{\prime} \in X$, $\alpha \delta_{x}+F \circ T_{A} \in \operatorname{ext} A^{*}$ and $\alpha^{\prime}, \beta^{\prime}$ are scalars then $x=x^{\prime}$ and $\alpha=\alpha^{\prime}$,

$\left(4_{\mathrm{C}}\right)$ if $\alpha \delta_{x}+\delta_{x} \circ T_{A}=\alpha^{\prime} \delta_{x^{\prime}}+\beta^{\prime} \delta_{x^{\prime}} \circ T_{A}$, where $x, x^{\prime} \in X, \alpha \delta_{x}+\delta_{x} \circ T_{A} \in \operatorname{ext} A^{*}$ and $\alpha^{\prime}, \beta^{\prime}$ are scalars, then $x=x^{\prime}$ and $\alpha=\alpha^{\prime}$.

\section{The results.}

THEOREM 1. Let $A$ and $B$ be $M$-subspaces of $C(X)$ and $C(Y)$, respectively. Put $X_{0}=\left\{x \in X: \delta_{x} \in \operatorname{ext} A^{*}\right\}, \tilde{X}=\left\{\alpha \delta_{x}: x \in X_{0}, \alpha \in S\right\}, Y_{0}=\left\{y \in Y: \delta_{y} \in\right.$ ext $\left.B^{*}\right\}, \tilde{Y}=\left\{\alpha \delta_{y}: y \in Y_{0}, \alpha \in S\right\}$. Then an isometry $\Phi$ from $A$ onto $B$ is canonical, of the form $(*)$ if and only if $\Phi^{*}(\tilde{Y})=\tilde{X}$.

The assumption of the above theorem, that $\Phi^{*}(\tilde{Y})=\tilde{X}$, looks very strong and so the theorem seems to be almost trivial, and as a matter of fact it is true, but the advantage of this statement is that for all the classical function spaces, with $M$ norm, this strong assumption can be easily verified. The method of verifying this, as we show in the next article, is the following. We define a property $P$ concerning the points of $A^{*}\left(B^{*}\right)$ such that $F \in A^{*}\left(G \in B^{*}\right)$ has this property if and only if $F \in \tilde{X}(G \in \tilde{Y})$. This property is defined by the weak-* topology of $A^{*}\left(B^{*}\right)$, the norms of $A$ and $A^{*}$ ( $B$ and $B^{*}$ ) and the linear structures of these Banach spaces. The map $\Phi^{*}$ is a weak- ${ }^{*}$ homeomorphism and a norm isometry from $B^{*}$ onto $A^{*}$ so $G \in B^{*}$ has the $P$ property if and only if $F=\Phi^{*}(G) \in A^{*}$ has the same property and hence we get $\Phi^{*}(\tilde{Y})=\tilde{X}$. A similar remark concerns the next theorems also.

ProOF. The "only if" part of Theorem 1 is trivial.

Assume that $\Phi^{*}(\tilde{Y})=\tilde{X}$. Then there are two functions $\varphi_{1}: \tilde{Y} \rightarrow X_{0}$ and $\varphi_{2}: \tilde{Y} \rightarrow S$ such that

$$
\Phi^{*}\left(\lambda \delta_{y}\right)=\varphi_{2}(\lambda, y) \cdot \delta_{\varphi_{1}(\lambda, y)} \quad \text { for } \lambda \delta_{y} \in \tilde{Y}
$$

For any $y \in Y$ and $\lambda_{1}, \lambda_{2} \in S$ functionals $\lambda_{1} \delta_{y}$ and $\lambda_{2} \delta_{y}$ are proportional. Hence $\delta_{\varphi_{1}\left(\lambda_{1}, y\right)}$ and $\delta_{\varphi_{1}\left(\lambda_{2}, y\right)}$ are also proportional and this means that $\varphi_{1}\left(\lambda_{1}, y\right)=$ $\varphi_{1}\left(\lambda_{2}, y\right)$. So $\varphi_{1}$ does not depend on the first coordinate and, by linearity of $\Phi^{*}$, the map $\varphi_{2}$ is linear with respect to $\lambda$. We get

$$
\Phi^{*}\left(\lambda \delta_{y}\right)=\lambda \varphi_{2}(y) \delta_{\varphi_{1}(y)} \text { for } \lambda \delta_{y} \in \tilde{Y} .
$$

Since $\Phi^{*}$ is a weak- ${ }^{*}$ homeomorphism of ext $B^{*}$ onto ext $A^{*}$ as well as a homeomorphism of the closures of these sets, and moreover the weak-* topologies of $\left\{\delta_{x}: x \in X\right\} \subset A^{*}$ and $\left\{\delta_{y}: y \in Y\right\} \subset B^{*}$ coincide with the original topologies of $X$ and $Y$, the map $\left(\varphi_{1}, \varphi_{2}\right): \tilde{Y} \rightarrow \tilde{X}$ can be extended to a homeomorphism $(\varphi, \chi)$ from $\overline{\tilde{Y}}=S \times Y$ onto $\overline{\tilde{X}}=S \times X$. Hence from (1) we get (*) and we are done. 
THEOREM 2. Let $A$ and $B$ be $\Sigma$-subspaces of $C(X)$ and $C(Y)$, respectively. Then an isometry $\Phi$ from $A$ onto $B$ is canonical, of the form (*), if and only if for any $\alpha_{i} \delta_{y_{i}}+G_{i} \circ T_{B} \in \operatorname{ext} B^{*}, i=1,2$, the following two implications hold:

(a) $y_{1}=y_{2}$ iff $x_{1}=x_{2}$ and

(b) $G_{1} \circ T_{B}$ and $G_{2} \circ T_{B}$ are proportional iff $F_{1} \circ T_{A}$ and $F_{2} \circ T_{A}$ are proportional; here by $x_{i}$ and $F_{i}$ we denote the elements of $X$ and ext $V^{*}$, respectively, such that $\Phi^{*}\left(\alpha_{i} \delta_{y_{i}}+G_{i} \circ T_{B}\right)=\beta_{i} \delta_{x_{i}}+F_{i} \circ T_{A}$, for some scalars $\beta_{i}, i=1,2$.

ProOF. As before the "only if" part is trivial. To prove the "if" part assume that an isometry $\Phi: A \rightarrow B$ satisfies our assumptions. Hence there are functions $\varphi_{1}, \varphi_{2}$ and $\varphi_{3}$ defined on the set ext $B^{*} \subset S \times Y \times \operatorname{ext} U^{*}$, with values in $X, S$ and $\operatorname{ext} V^{*}$, respectively, such that

$$
\begin{array}{r}
\Phi^{*}\left(\alpha \delta_{y}+G \circ T_{B}\right)=\varphi_{2}(\alpha, y, G) \delta_{\varphi_{1}(\alpha, y, G)}+\varphi_{3}(\alpha, y, G) \circ T_{A} \\
\text { for } \alpha \delta_{y}+G \circ T_{B} \in \operatorname{ext} B^{*} .
\end{array}
$$

By the assumption (a) the function $\varphi_{1}$ does not depend on $\alpha$ and $G$ and we write $\varphi_{1}(y)$ in place of $\varphi_{1}(\alpha, y, G)$. By (b) the map $\varphi_{3}$ is of the form $\varphi_{3}(\alpha, y, G)=$ $\psi(\alpha, y) \varphi_{4}(G)$ where $\varphi_{4}(G) \in \operatorname{ext} V^{*}$ and $\psi$ has values in $S$.

By the same argument as in the proof of Theorem 1 the map $\varphi_{1}$ can be extended to a homeomorphism $\varphi$ from $Y$ onto $X$. So to end the proof we have to show that $\Phi^{*}$ maps $\alpha \delta_{y}$ onto $\varphi_{2}(\alpha, y, G) \delta_{\varphi(y)}$.

For any $H=\alpha \delta_{y}+G \circ T_{B} \in \operatorname{ext} B^{*}$ we define

$$
\Omega_{B}(H)=\left\{\alpha^{\prime} \delta_{y}+\beta^{\prime} G \circ T_{B} \in \operatorname{ext} B^{*}: \alpha^{\prime}, \beta^{\prime} \in S\right\} .
$$

By our assumptions we have $\Omega_{B}(H)=\Omega_{A}\left(\Phi^{*}(H)\right)$ for any $H \in \operatorname{ext} B^{*}$. Let $G_{0}$ and $Y_{0}$ be as in assumption $\left(3_{\Sigma}\right)$ for the space $B$. Notice that for any $y \in Y_{0}$ the set $\Omega_{B}\left(\delta_{y}+G_{0} \circ T_{B}\right)$ is homeomorphic to $S \times S$. Hence, by (2), the set $\Omega_{A}\left(\varphi_{2}\left(1, y, G_{0}\right) \delta_{\varphi_{1}(y)}+\psi(1, y) \varphi_{4}\left(G_{0}\right)\right)$ is also homeomorphic to $S \times S$ and so

$$
\alpha \delta_{\varphi_{1}(y)}+\beta \varphi_{4}\left(G_{0}\right) \in \operatorname{ext} A^{*} \quad \text { for all } y \in Y_{0}, \alpha, \beta \in S .
$$

Hence we have $X_{0}=\varphi_{1}\left(Y_{0}\right)$ and we can put $F_{0}=\varphi_{4}\left(G_{0}\right)$ in our assumption $\left(3_{\Sigma}\right)$.

Notice that for any vector space $E$, for any functionals $e_{1}^{*}, e_{2}^{*}$ on $E$ and for any $e \in E$ we have

$$
\operatorname{card}\left(\left\{\left|\alpha e_{1}^{*}(e)+\beta e_{2}^{*}(e)\right|: \alpha, \beta \in S\right\}\right)=1 \quad \text { iff } \quad e_{1}^{*}(e) \cdot e_{2}^{*}(e)=0 .
$$

Hence for any $f \in A$ and any $y \in Y_{0}$ we have the following implications:

$$
\begin{aligned}
& f\left(\varphi_{1}(y)\right)=0 \text { or } F_{0} \circ T_{A}(f)=0 \quad \text { iff } \\
& \operatorname{card}\left(\left\{|H(f)|: H \in \Omega_{A}\left(\delta_{\varphi_{1}(y)}+F_{0} \circ T_{A}\right)\right\}\right)=1 \quad \text { iff } \\
& \operatorname{card}\left(\left\{|H(\Phi(f))|: H \in \Omega_{B}\left(\delta_{y}+G_{0} \circ T_{B}\right)\right\}\right)=1 \quad \text { iff } \\
& \Phi(f)(y)=0 \text { or } G_{0} \circ T_{B}(\Phi(f))=0 .
\end{aligned}
$$

This means that the union of $\operatorname{ker} \delta_{\varphi_{1}(y)}$ and $\operatorname{ker} F_{0} \circ T_{A}$ is equal to the union of $\operatorname{ker} \Phi^{*}\left(\delta_{y}\right)$ and $\operatorname{ker} \Phi^{*}\left(G_{0} \circ T_{B}\right)$. By the assumption $\left(4_{\Sigma}\right)$ functionals $\delta_{\varphi_{1}(y)}$ and $F_{0} \circ T_{A}$ are linearly independent so the above proves that for any $y \in Y_{0}$ we have two possibilities. 
(i) $\Phi^{*}\left(\delta_{y}\right)=\chi(y) \delta_{\varphi_{1}(y)}$ and $\Phi^{*}\left(G_{0} \circ T_{B}\right)=\chi^{\prime}(y) F_{0} \circ T_{A}$ or

(ii) $\Phi^{*}\left(\delta_{y}\right)=\chi^{\prime}(y) F_{0} \circ T_{A}$ and $\Phi^{*}\left(G_{0} \circ T_{B}\right)=\chi(y) \delta_{\varphi_{1}(y)}$, where $\chi$ and $\chi^{\prime}$ are scalar valued functions.

On the other hand by our assumption (b) we have

$\Phi^{*}\left(\left\{\alpha \delta_{y}+\beta G_{0} \circ T_{B}: y \in Y, \alpha, \beta \in S\right\}\right)=\left\{\alpha \delta_{x}+\beta F_{0} \circ T_{A}: x \in X, \alpha, \beta \in S\right\}$.

This shows that the second possibility does not hold for any $y \in Y_{0}$ (except in the trivial case when $\operatorname{card}(Y)=1)$; so to end the proof of $(*)$ it is sufficient to notice that by (i), (2) and by the assumption $\left(4_{\Sigma}\right)$ we have $|\chi|=\left|\varphi_{2}\right| \equiv 1$.

THEOREM 3. Let $A$ and $B$ be $C$-subspaces of $C(X)$ and $C(Y)$, respectively. Then an isometry $\Phi$ from $A$ onto $B$ is canonical, of the form (*), if and only if the following two conditions hold:

(a) for any $\alpha_{i} \delta_{y_{i}}+\beta_{i} \delta_{y_{i}} \circ T_{B} \in \operatorname{ext} B^{*}, i=1,2$, we have $y_{1}=y_{2}$ iff $x_{1}=x_{2}$, where by $x_{i}$ we denote an element of $X$ such that

$$
\Phi^{*}\left(\alpha_{i} \delta_{y_{i}}+\beta_{i} \delta_{y_{i}} \circ T_{B}\right)=\alpha_{i}^{\prime} \delta_{x_{i}}+\beta_{i}^{\prime} \delta_{x_{i}} \circ T_{A},
$$

for some scalars $\alpha_{i}^{\prime}, \beta_{i}^{\prime}, i=1,2$;

(b) $\Phi^{*}\left(\left\{\alpha \delta_{y}: y \in Y, \alpha \in S\right\}\right) \cap\left\{\alpha \delta_{x} \circ T_{A}: x \in X, \alpha \in S\right\}=\varnothing$.

PrOOF. The "only if" part is again trivial. Assume that $\Phi$ satisfies assumptions (a) and (b). Following what is by now a standard argument we get a homeomorphism $\varphi$ from $Y$ onto $X$ and scalar valued functions $\varphi_{2}$ and $\varphi_{3}$ defined on $S \times S \times Y$, with $\left|\varphi_{2}\right| \equiv 1 \equiv\left|\varphi_{3}\right|$ such that

$$
\Phi^{*}\left(\alpha \delta_{y}+\beta \delta_{y} \circ T_{B}\right)=\varphi_{2}(\alpha, \beta, y) \delta_{\varphi(y)}+\varphi_{3}(\alpha, \beta, y) \cdot \delta_{\varphi(y)} \circ T_{A},
$$

where $y \in Y$ and $\alpha, \beta \in S$. For any $H=\alpha \delta_{x}+\beta \delta_{x} \circ T_{A} \in \operatorname{ext} A^{*}$ we define

$$
\Omega_{A}(H)=\left\{\alpha^{\prime} \delta_{x}+\beta^{\prime} \delta_{x} \circ T_{A} \in \operatorname{ext} A^{*}: \alpha, \beta \in S\right\} .
$$

As in the proof of the preceding theorem we show that for any $y \in Y$ we have two possibilities:

$$
\begin{gathered}
\Phi^{*}\left(\delta_{y}\right)=\chi(y) \delta_{\varphi(y)} \quad \text { and } \quad \Phi^{*}\left(\delta_{y} \circ T_{B}\right)=\chi^{\prime}(y) \cdot \delta_{\varphi(y)} \circ T_{A} \quad \text { or } \\
\Phi^{*}\left(\delta_{y}\right)=\chi^{\prime}(y) \delta_{\varphi(y)} \circ T_{A} \quad \text { and } \quad \Phi^{*}\left(\delta_{y} \circ T_{B}\right)=\chi(y) \delta_{\varphi(y)} .
\end{gathered}
$$

By the assumption (b) the second possibility never holds; by $\left(4_{C}\right)$ we then get $|\chi| \equiv\left|\varphi_{2}\right| \equiv 1$ and we are done.

To verify the assumptions of our schemes, given by Theorems $1-3$, it is usually necessary to have at least partial description of the extreme functionals in the unit ball of the dual spaces. Hence in some cases it is easier to apply the following theorem, which is an immediate consequence of the Theorem of [12].

THEOREM 4. Let $A$ be a complex subspace of $C(X), X$ compact Hausdorff space, such that

(i) $A$ is sup-norm dense in $C(X)$,

(ii) the norm on $A$ is given by a map $T_{A}: A \rightarrow V_{A}$, via the formula $(\mathrm{M})$ or $(\Sigma)$,

(iii) $A$ contains the constant function 1 and $T_{A}(\mathbf{1})=0$. 
Assume next that $B$ is a complex subspace of $C(Y)$ which satisfies the analogous assumptions (i)-(iii). Then any isometry $\Phi$ from $A$ onto $B$, such that $\Phi(\mathbf{1})=1$ is of the form

$$
\Phi(f)=f \circ \varphi \text { for } f \in A,
$$

where $\varphi$ is a homeomorphism from $Y$ onto $X$.

\section{Examples.}

EXAMPLE 1. Let $A=C^{1}(X)$ and $B=C^{1}(Y)$ be algebras of continuously differentiable functions defined on the compact subsets $X$ and $Y$ of the real line, respectively. We do not assume here that the sets $X$ and $Y$ do not contain isolated points but we understand that the derivative of a function $f \in C^{1}(X)$ is defined only on the set of nonisolated points of $X$. Assume that the norms on $A$ and $B$ are given by the formula $(\mathrm{M})$; this means

$$
\|f\|=\max \left(\|f\|_{\infty},\left\|f^{\prime}\right\|_{\infty}\right), \quad f \in A(B) .
$$

We prove that $A$ is an $M$-subspace of $C(X)$. The first two assumptions of the definition of $M$-subspace are evidently fulfilled. To show the last one notice that for any $x_{0} \in X$ there is an $f \in C^{1}(X)$ such that $\|f\|=\|f\|_{\infty}=f\left(x_{0}\right)=1>\left\|f^{\prime}\right\|_{\infty}$ and such that $|f(x)|<1$ for $x \in X \backslash\left\{x_{0}\right\}$. Hence for any $x_{0} \in X$ the functional $\delta_{x_{0}}$ is an extreme point of $A_{1}^{*}$, so $X_{0}=X$. Notice also that for any $x_{0} \in X$ there is a $g \in C^{1}(X)$ such that $\|g\|=\left\|g^{\prime}\right\|_{\infty}=g^{\prime}\left(x_{0}\right)=1>\|g\|_{\infty}$ and such that $\left|g^{\prime}(x)\right|<1$ for $x \in X \backslash\left\{x_{0}\right\}$. Hence

$$
\text { ext } A^{*}=\left\{a \delta_{x}: x \in X, \alpha \in S\right\} \cup\left\{\alpha \delta_{x}^{\prime}: x \in X, \alpha \in S\right\},
$$

where by $\delta_{x}^{\prime}$ we denote a functional defined by $\delta_{x}^{\prime}(f)=\delta_{x} \circ D(f)=f^{\prime}(x)$.

Now let $\Phi$ be any isometry from $A$ onto $B$. We prove that $\Phi^{*}(\tilde{Y})=\tilde{X}$. To this end we define the following property concerning points of $A^{*}\left(B^{*}\right)$.

$F \in A^{*}$ has the $P$-property iff $F \in \operatorname{ext} A^{*}$ and there is a $G$ in ext $A^{*}$, not proportional to $F$, such that for any weak-* open neighborhood $U \subset \operatorname{ext} A^{*}$ of $G$ and any $f \in A \subset A^{* *}$ we have: if $\left.f\right|_{U} \equiv 0$ then $F(f)=0$.

We check that $F \in A^{*}$ has the $P$-property if and only if $F \in \operatorname{ext} A^{*} \backslash \tilde{X}$. Fix any point $x_{0} \in X$ and put $F=\alpha \delta_{x_{0}}^{\prime}$ with $\alpha \in S$. By (4) $F \in \operatorname{ext} A^{*}$ and for any weak-* open neighborhood $U \subset \operatorname{ext} A^{*}$ of the functional $\delta_{x_{0}}$ and for any $f \in A$ we have

$$
\begin{aligned}
\left.f\right|_{U} \equiv 0 & \Rightarrow f \equiv 0 \text { on an open neighborhood of } x_{0} \\
& \Rightarrow f^{\prime}\left(x_{0}\right)=0 .
\end{aligned}
$$

Hence any element of $\operatorname{ext} A^{*} \backslash \tilde{X}$ has the $P$-property.

Now fix again $x_{0} \in X$ and put $F=\alpha \delta_{x_{0}}$. Let $G \in \operatorname{ext} A^{*} \backslash\{\gamma F: \gamma \in S\}$. By (4) we have two possibilities: $1 . G=\beta \delta_{x_{1}}, x_{1} \neq x_{0}$ or $2 . G=\beta \delta_{x_{1}}^{\prime}$. If the first one holds then we put $U=\left\{\gamma \delta_{x}:\left|x-x_{1}\right|<\left|x_{0}-x_{1}\right| / 2, \gamma \in S\right\}$; evidently there is an $f$ in $A \subset A^{* *}$ such that $\left.f\right|_{U} \equiv 0$ and $f\left(x_{0}\right)=1$. If the second possibility holds we put $U=\left\{\gamma \delta_{x}^{\prime}: x \in X, \gamma \in S\right\}$ and put $f \equiv 1$. Hence $F$ does not have the $\mathcal{P}$-property.

We have proved that $F \in A^{*}$ has the $P$-property if and only if $F \in \operatorname{ext} A^{*} \backslash \tilde{X}$. This property is defined by the weak-* topology of $A^{*}$, and the norm and the linear structure of $A^{*}$; this means by the properties which are preserved by $\Phi^{*}$. Hence 
$F=\Phi^{*}(G) \in A^{*}$ has this property if and only if $G \in B^{*}$ has, and this proves that $\Phi^{*}(\tilde{Y})=\tilde{X}$.

By Theorem 1 we now get that any isometry $\Phi$ from $C^{1}(X)$ onto $C^{1}(Y)$ is of the form

$$
\Phi(f)(y)=\chi(y) \cdot f \cdot \varphi(y) \text { for } f \in A, y \in Y,
$$

where $\chi \in C^{1}(X),|\chi| \equiv 1$ and $\varphi$ is a homeomorphism from $Y$ onto $X$. It is also easy to verify now that since $\Phi$ preserves both $M$-norm and sup-norm, it also preserves the sup-norm of the derivative and hence we get $\left|\varphi^{\prime}\right| \equiv 1$.

We have assumed at the beginning of this example that the sets $X$ and $Y$ are compact. In fact this assumption is not essential and the same holds for arbitrary subsets of the real line not necessarily bounded and closed. We then consider $A$ as a subset of $C(\beta X)$ and the proof is slightly more technical.

The general form of the isometries of complex $C^{1}(X)$ spaces, defined on a compact subset $X$ of the real line, without isolated points, was investigated by Pathak and Vasavada [19].

EXAMPLE 2. Let $A=\mathrm{AC}(X)$ be the space of all absolutely continuous, scalar valued functions defined on a compact subset $X$ of the real line, such that $X=\overline{\operatorname{int} X}$. We define norm on $A$ by

$$
\|f\|=\max \left(\|f\|_{\infty},\left\|f^{\prime}\right\|_{1}\right) \text { for } f \in A
$$

where $\left\|f^{\prime}\right\|_{1}=\int_{X}\left|f^{\prime}\right| d m$ and $m$ is the Lebesgue measure.

As in the preceding example it is easy to notice that for any $x_{0} \in X$ there is an $f \in A$ such that $\|f\|=\|f\|_{\infty}=f\left(x_{0}\right)=1>\left\|f^{\prime}\right\|_{1}$ and $|f(x)|<1$ for $x \in X \backslash\left\{x_{0}\right\}$. Hence $X_{0}=X$ and $A$ is an $M$-subspace of $C(X)$. Notice also that for any $F \in \operatorname{ext}\left(L^{1}(m)\right)^{*} \cong \operatorname{ext}\left(L^{\infty}(m)\right)$ and a function $f \in \mathrm{AC}(X)$ defined by

$$
f(t)=\frac{1}{m(X)} \int_{X \cap(-\infty, t)} \bar{F}(x) d m(x)-\frac{1}{2 m(X)} \int_{X} \bar{F}(x) d m(x)
$$

we have $\|f\|=1,\|f\|_{\infty} \leq \frac{1}{2}$ and for any $G \in \operatorname{ext}\left(L^{\infty}\right)=\operatorname{ext}\left(L^{1}\right)$

$$
G(f):=\int_{X} G f^{\prime} d m=1 \quad \text { iff } G=F .
$$

This means that the function $f \in A \subset C\left(X \cup \operatorname{ext}\left(L^{\infty}\right)\right)$ peaks exactly at the point $F \in \operatorname{ext}\left(L^{\infty}\right)$, so any such point $F$ is an extreme point of $A^{*}$ and we have

$$
\operatorname{ext} A^{*}=\left\{\alpha \delta_{x}: x \in X, \alpha \in S\right\} \cup\left\{F \circ D: F \in \operatorname{ext}\left(L^{\infty}\right)\right\},
$$

where $D: \operatorname{AC}(X) \rightarrow L^{1}$ is defined by $D(f)=f^{\prime}$.

By Theorem 1 to prove that any isometry $\Phi$ from $\mathrm{AC}(X)$ onto $\mathrm{AC}(Y)$ is canonical we have to, as before, define the set $\tilde{X}=\left\{\alpha \delta_{x} \in \operatorname{ext} A^{*}: x \in X, \alpha \in S\right\}$ by the weak-* topology of $A^{*}$ and the norms and the linear structures of $A$ and $A^{*}$. We have

$F \in \tilde{X}$ iff $F \in \operatorname{ext} A^{*}$ and there is a weak-* open neighborhood of $F$ in ext $A^{*}$ which is homeomorphic to a subset of $S \times \mathbf{R}, \mathbf{R}$ the real line. 
EXAMPLE 3. If $X$ is any compact metric space with metric $d$ we let

$$
\operatorname{Lip}_{\alpha}(X)=\left\{f \in C(X):\|f\|_{d^{\alpha}}=\sup _{\substack{x, y \in Y \\ x \neq y}} \frac{|f(x)-f(y)|}{d^{\alpha}(x, y)}<\infty\right\}
$$

and

$$
\operatorname{lip}_{\alpha}(X)=\left\{f \in \operatorname{Lip}_{\alpha}(X): \lim _{d(x, y) \rightarrow 0} \frac{f(x)-f(y)}{d^{\alpha}(x, y)}=0\right\},
$$

both provided with the $M$-norm; this means with the norm defined by

$$
\|f\|=\max \left(\|f\|_{\infty},\|f\|_{d^{\alpha}}\right) .
$$

As before it is easy to check that both $\operatorname{Lip}_{\alpha}(X)$ and $\operatorname{lip}_{\alpha}(X)$ are $M$-subspaces of $C(X)$. Therefore to prove that any isometry from $\operatorname{Lip}_{\alpha}(X)$ onto $\operatorname{Lip}_{\alpha}(Y)$ or from $\operatorname{lip}_{\alpha}(X)$ onto $\operatorname{lip}_{\alpha}(Y)$ is canonical we have to define a property which "separates" $\tilde{X}=\left\{\alpha \delta_{x}: x \in X, \alpha \in S\right\}$ from the rest of the extreme points of the unit ball of $\left(\operatorname{Lip}_{\alpha}(X)\right)^{*}$ or $\left(\operatorname{lip}_{\alpha}(X)\right)^{*}$. We have

$F \in \operatorname{ext} A^{*} \backslash \tilde{X}$ iff $F \in \operatorname{ext} A^{*}$ and there are sequences $\left(F_{1}^{n}\right)_{n=1}^{\infty}$ and $\left(F_{2}^{n}\right)_{n=1}^{\infty}$ such that $\left(F_{1}^{n}-F_{2}^{n}\right) /\left\|F_{1}^{n}-F_{2}^{n}\right\|$ tends to $F$ in the norm topology.

The isometries of the complex $\operatorname{Lip}_{1}(X)$ spaces with the above $M$-norm were considered by Roy [21], when $X$ is connected with diameter at most 1 , and by Vasavada [22], when $X$ satisfies certain separation conditions. A similar space defined on the real line was investigated by de Leeuw [15].

EXAMPLE 4. Let $A=C^{1}(X)$ and $B=C^{1}(Y)$ be the same vector spaces as in Example 1 but now with the $\Sigma$-norm; this means with the norm given by

$$
\|f\|=\|f\|_{\infty}+\left\|f^{\prime}\right\|_{\infty}, \quad f \in A(B) .
$$

To prove that $A$ is a $\Sigma$-subspace of $C(X)$ it is sufficient to notice that for any $x_{1} \neq x_{2}$ in $X$ and any $\alpha, \beta \in S$ there is an $f$ in $A$ such that $\|f\|=1, f\left(x_{1}\right)=\bar{\alpha} / 2$, $|f(x)|<\frac{1}{2}$ for $x \neq x_{1}, f^{\prime}\left(x_{2}\right)=\bar{\beta} / 2$ and $\left|f^{\prime}(x)\right|<\frac{1}{2}$ for $x \neq x_{2}$. Hence

$$
\left\{\alpha \delta_{x}+\beta \delta_{y} \circ D: x \neq y \in X, \alpha, \beta \in S\right\} \subset \operatorname{ext} A^{*} .
$$

Now let $\Phi$ be any isometry from $A$ onto $B$. To prove that $\Phi$ is canonical, by Theorem 2, we have to show that the assumptions (a) and (b) of this theorem are satisfied. To see this we define two equivalence relations $\sim_{1}$ and $\sim_{2}$ on ext $A^{*}$ by the following formulas:

$$
\alpha_{1} \delta_{x_{1}}+F_{1} \circ D \sim_{1} \alpha_{2} \delta_{x_{2}}+F_{2} \circ D \text { iff } x_{1}=x_{2}
$$

and

(6) $\alpha_{1} \delta_{x_{1}}+F_{1} \circ D \sim_{2} \alpha_{2} \delta_{x_{2}}+F_{2} \circ D$ iff $F_{1} \circ D$ and $F_{2} \circ D$ are proportional.

To verify (a) and (b) we just have to prove that the map $\Phi^{*}$ preserves both the above relations. We prove this by defining $\sim_{1}$ and $\sim_{2}$ in terms of the weak-* topology of $A^{*}$, the norms and the linear structures of $A$ and $A^{*}$, so in the terms which are preserved by $\Phi^{*}$. To this end we notice that a sequence $\left(\alpha_{n} \delta_{x_{n}}+\beta_{n} \delta_{y_{n}} \circ D\right)_{n=1}^{\infty} \subset$ ext $A^{*}$ tends to $\alpha_{0} \delta_{x_{0}}+\beta_{0} \delta_{y_{0}} \circ D$ in the weak-* topology iff $\alpha_{n} \rightarrow \alpha_{0}, \beta_{n} \rightarrow \beta_{0}$, $x_{n} \rightarrow x_{0}$ and $y_{n} \rightarrow y_{0}$; and in the norm topology iff $\alpha_{n} \rightarrow \alpha_{0}, \beta_{n} \rightarrow \beta_{0}, x_{n} \rightarrow x_{0}$ 
and $y_{n}=y_{0}$ for all but finitely many $n$. We have

$F \sim_{1} G$ iff there are sequences $\left(F_{n}\right)_{n=1}^{\infty}$ and $\left(G_{n}\right)_{n=1}^{\infty}$ in ext $A^{*}$ which tend, in the norm topology, to $F$ and $G$, respectively and there is a scalar $\alpha$ such that $\operatorname{dim}\left(\operatorname{span}\left\{F_{n}: n=1,2, \ldots\right\}\right)=\infty$ and $F_{n}+\alpha G_{n}=F+\alpha G$ for all $n \in \mathbf{N}$.

We also have

$F \sim_{2} G$ iff there are sequences $\left(F_{n}\right)_{n=1}^{\infty}$ and $\left(G_{n}\right)_{n=1}^{\infty}$ in ext $A^{*}$ which tend to $F$ and $G$ respectively, in the weak-* topology, but do not tend in the norm topology, and there is a scalar $\alpha$ such that $F_{n}+\alpha G_{n}=F+\alpha G$ for all $n \in \mathbf{N}$.

The isometries of the complex $C^{1}[0,1]$ space with the above $\Sigma$-norm were described by Rao and Roy [20].

EXAMPLE 5 . Let $A=\mathrm{AC}[0,1]$ be the spaces of all complex absolutely continuous functions defined on the unit interval with the $\Sigma$-norm; this means with the norm given by

$$
\|f\|=\|f\|_{\infty}+\left\|f^{\prime}\right\|_{1}, \quad f \in A
$$

As we noticed in $\S 2$ any extreme functional on $A$ is of the form

$$
A \ni f \stackrel{\alpha \delta_{x}+F \circ D}{\longmapsto} \alpha f(x)+\int F \cdot f^{\prime} d m,
$$

where $x \in X, \alpha \in S, m$ is the Lebesgue measure and $F$ is an extreme point of $\left(L^{1}\right)^{*}$, which we identify with a function $F \in L^{\infty}$ such that $|F|=1$ a.e. To prove that $A$ is a $\Sigma$-subspace of $C[0,1]$ we have to show that there is an $F_{0}$ in $\operatorname{ext}\left(L^{\infty}\right)$ such that for any $x \in X$ and any $\alpha \in S$ the corresponding functional defined by (7) is an extreme point of $A_{1}^{*}$. To this end let $K$ be a measurable subset of $[0,1]$ such that for any open subset $U$ of $[0,1]$ we have $0<m(K \cap U)<m(U)$. By Lemma 2.1 of $[20]$ the desired function $F_{0}$ can be defined by

$$
F_{0}(t)= \begin{cases}1 & \text { if } t \in K, \\ -1 & \text { if } t \notin K .\end{cases}
$$

To prove that any isometry from $A$ onto itself is canonical we have to, as in the previous example, describe the equivalence relations defined by (5) and (6) by the weak-* topology of $A^{*}$, the norms and the linear structures of $A$ and $A^{*}$. To this end notice that a sequence $\left(\alpha_{n} \delta_{x_{n}}+F_{n} \circ D\right)_{n=1}^{\infty} \subset$ ext $A^{*}$ tends to $\alpha \delta_{x}+F \circ D$ in the weak-* topology iff $\alpha_{n} \rightarrow \alpha, x_{n} \rightarrow x$ and $F_{n}$ tends to $F$ in the weak-* topology of $L^{\infty}$; and in the norm topology iff $\alpha_{n} \rightarrow \alpha, x_{n}=x$ for all but finitely many $n \in \mathrm{N}$ and $\left\|F_{n}-F\right\| \rightarrow 0$.

By Lemma 2.1 of $[20]$ we have

$F \sim_{1} G$ iff $F$ and $G$ are contained in the same connected component of the set ext $A^{*}$ equipped with the norm topology,

and

$F \sim_{2} G$ iff there are sequences $\left(F_{n}\right)_{n=1}^{\infty}$ and $\left(G_{n}\right)_{n=1}^{\infty}$ in ext $A^{*}$ which tend, in the norm topology, to $F$ and $G$, respectively and a scalar $\alpha$ such that $\operatorname{dim}\left(\operatorname{span}\left\{F_{n}: n=1,2, \ldots\right\}\right)=\infty$ and $F_{n}+$ $\alpha G_{n}=F+\alpha G$ for all $n \in \mathbf{N}$. 
The isometries of the spaces $\mathrm{AC}[0,1]$ have been described by Cambern $[3]$ and by Rao and Roy [20].

EXAMPLE 6 . Let $A=C^{1}(X)$ and $B=C^{1}(Y)$ be as in Example 1 but now with the $C$-norm; this means with the norm given by

$$
\|f\|=\sup \left\{|f(x)|+\left|f^{\prime}(x)\right|: x \in X\right\} .
$$

In order for $\|\cdot\|$ to be a well-defined norm we have to assume now that $X$ and $Y$ do not contain isolated points.

As before, it is standard to verify (see [5, Lemma 2], for the complex case) that

$$
\operatorname{ext} A^{*}=\left\{\alpha \delta_{x}+\beta \delta_{x} \circ D: x \in X, \alpha, \beta \in S\right\} \text {. }
$$

Hence $A$ and $B$ are $C$-subspaces of $C(X)$ and $C(Y)$, respectively. To prove that any isometry from $A$ onto $B$ is canonical we have to check whether assumptions (a) and (b) of Theorem 3 are satisfied. To this end we describe the equivalence relation on $\operatorname{ext} A^{*}$ defined by

$$
\alpha \delta_{x}+\beta \delta_{x} \circ D \sim \alpha^{\prime} \delta_{x^{\prime}}+\beta^{\prime} \delta_{x^{\prime}} \circ D \text { iff } x=x^{\prime}
$$

in terms of the weak-* topology of $A^{*}$, the norms and the linear structures of $A$ and $A^{*}$, and we also "separate" the set $\left\{\alpha \delta_{x} \in A^{*}: x \in X, \alpha \in S\right\}$ from the set $\left\{\alpha \delta_{x} \circ D \in A^{*}: x \in X, \alpha \in S\right\}$ by the same properties, which are preserved by $\Phi^{*}$. We have

$F \sim G$ iff $F$ and $G$ are contained in the same connected component of the set ext $A^{*}$, equipped with the norm topology.

We also have

$F \in\left\{\alpha \delta_{x}: x \in X, \alpha \in S\right\}$ iff $\{\alpha F: \alpha \in S\}$ is an open subset of the set $\left\{\alpha \delta_{x}: x \in X, \alpha \in S\right\} \cup\left\{\alpha \delta_{x} \circ D: x \in X, \alpha \in S\right\}$, equipped with the norm topology.

The isometries of the complex $C^{1}(X)$ spaces with the $C$-norm have first been considered by Cambern [3] for $X=[0,1]$ and then by Cambern and Pathak [5] for $X$ any compact subset of the real line, without isolated points.

REMARK. Following the same arguments as given in Examples 1, 4, 6 it can be shown that for a much wider class of $C^{1}(K)$-type algebras, with $M-, \Sigma$ - or $C$-norm, any isometry is canonical. For example let $K$ be a compact subset of the complex plane with connected complement. Let $A(K) \subset C(K)$ be the sup-norm closure of the space of all polynomials and put

$$
A^{1}(K)=\left\{f \in A(K): D(f)=f^{\prime} \in A(K)\right\} .
$$

It can be proved that $A^{1}(K)$ gives $M$-, $\Sigma$ - and $C$-subspaces of $C(\partial K)$, where $\partial K$ is the topological boundary of $K$, and that all the assumptions of Theorems 1,2 and 3 are satisfied for $A=A^{1}(K), B=A^{1}(L)$. Hence any $M$-, $\Sigma$ - or $C$-isometry $\Phi$ from $A^{1}(K)$ onto $A^{1}(L)$ is of the form

$$
\Phi f(z)=\chi(z) \cdot f \circ \varphi(z), \quad f \in A^{1}(K), z \in L,
$$

where $\varphi$ is an analytic homeomorphism from $L$ onto $K$, with $\left|\varphi^{\prime}\right| \equiv 1$ and $\chi \in$ $A^{1}(K)$, with $|\chi| \equiv 1$. 
EXAMPLE 7. Let $X$ be a compact subset of the real line. For $1 \leq p \leq \infty$ we define

$$
\operatorname{AC}^{p}(X)=\left\{f \in C(X): f^{\prime} \text { exists a.e., } f^{\prime} \in L^{p}(X)\right\}
$$

and we define a norm on $\mathrm{AC}^{p}(X)$ by

$$
\|f\|=\|f\|_{\infty}+\left\|f^{\prime}\right\|_{p} .
$$

For $p=1$ (resp. $\infty$ ) we get the space $\operatorname{AC}(X)(\operatorname{resp} . \operatorname{Lip}(X))$. Rao and Roy [20] proved that any isometry from the complex $\operatorname{AC}^{p}([0,1])$ space, $p=1$ or $\infty$, onto itself is canonical (cf. Example 5) and asked whether the same holds for $1<p<\infty$. The answer is positive and is a consequence of the following more general proposition.

Proposition. Let $A$ be a complex subspace of $C(X), X$ compact Hausdorff space, such that

(i) $A$ is sup-norm dense in $C(X)$,

(ii) the norm on $A$ is given by a map $T_{A}: A \rightarrow V_{A}$, via the formula $(\Sigma)$,

(iii) $A$ contains the constant function 1 and $T_{A}(\mathbf{1})=0$,

(iv) $\operatorname{dim}\left(T_{A}(A)\right) \geq 2$,

(v) $V$ is strictly convex,

(iv) for any unimodular function $\chi \in A$ such that $T_{A}(\chi)=0$ a map

$$
A \ni f \mapsto f / \chi \in A
$$

is a well-defined surjective isometry.

Assume next that $B$ is a complex subspace of $C(Y), Y$ compact Hausdorff, which also satisfies assumptions (i)-(vi). Then any isometry $\Phi$ from $A$ onto $B$ is of the form

$$
\Phi(f)=\chi \cdot f \circ \varphi, \quad f \in A,
$$

where $\varphi$ is a homeomorphism from $Y$ onto $X$ and $\chi \in B$ is a unimodular function on $Y$ such that $T_{B}(\chi)=0$.

Proof of THE Proposition. By Theorem 4 and by our assumption (vi) it is sufficient to prove that $\Phi(1)$ is a unimodular function on $Y$ such that $T_{B}(\Phi(1))=0$.

We say that an element $g$ of $A$ has the $P$-property if $\|g\|=1$ and if for any $f$ in $A$ there is a $\beta \in S$ such that

$$
\|g+\beta f\|=\|g\|+\|f\| .
$$

It is evident that this property is preserved by our isometry $\Phi$. By the definition of the norm on $A$ for any $f \in A$ and $\beta \in S$ such that $\|f\|_{\infty}=\sup _{x \in X} \operatorname{Re}(\beta f(x))$ we have

$$
\|1+\beta f\|=\|1+\beta f\|_{\infty}+\left\|T_{A}(f)\right\|=1+\|f\|_{\infty}+\left\|T_{A}(f)\right\|=1+\|f\| .
$$

Hence to end the proof we have to show that if $g \in A$ has the property $P$ then $g$ is a unimodular function on $X$ such that $T_{A}(g)=0$.

We first prove that $|g| \equiv c$ on $X$ for some constant $c$, then we prove that $T_{A}(g)=$ 0 and then from the definition of the norm on $A$ we get $c=\|g\|_{\infty}=\|g\|=1$.

Assume that there is an $x_{0} \in X$ such that $\left|g\left(x_{0}\right)\right|<\|g\|_{\infty}$. By assumption (i) there is an $f$ in $A$ such that

$$
\|f\|_{\infty}=\|g\|_{\infty}-\left|g\left(x_{0}\right)\right|
$$


and

$$
|f(x)| \leq\|g\|_{\infty}-|g(x)|+\frac{1}{2}\left(\|g\|_{\infty}-\left|g\left(x_{0}\right)\right|\right) \quad \text { for } x \in X .
$$

For any $\beta \in S$ we have

$$
\|g+\beta f\|_{\infty} \leq\|g\|_{\infty}+\frac{1}{2}\|f\|_{\infty}
$$

so

$$
\begin{aligned}
\|g+\beta f\| & =\|g+\beta f\|_{\infty}+\left\|T_{A}(g+\beta f)\right\| \\
& \leq\|g\|_{\infty}+\frac{1}{2}\|f\|_{\infty}+\left\|T_{A}(g)\right\|+\left\|T_{A}(f)\right\| \\
& =\|g\|+\|f\|-\frac{1}{2}\|f\|_{\infty}<1+\|f\| .
\end{aligned}
$$

Hence $|g|=$ const. Assume now that $T_{A}(g) \neq 0$; by assumption (iv) there is an $f$ in $A$ such that $T_{A}(f)$ and $T_{A}(g)$ are not proportional. Since $V_{A}$ is strictly convex for any $h, h^{\prime}$ in $V_{A}$ we have

$$
\left\|h+h^{\prime}\right\|=\|h\|+\left\|h^{\prime}\right\| \quad \text { iff } \quad h \text { and } h^{\prime} \text { are proportional }
$$

and hence for any $\beta \in S$ we get

$$
\begin{aligned}
\|g+\beta f\| & =\|g+\beta f\|_{\infty}+\left\|T_{A}(g+\beta f)\right\| \\
& <\|g\|_{\infty}+\|f\|_{\infty}+\left\|T_{A}(g)\right\|+\left\|T_{A}(f)\right\|=1+\|f\| .
\end{aligned}
$$

Hence $T_{A}(g)=0$ and we are done.

EXAMPLE 8 . Let $X$ be a compact metric space, with metric $d$ and let $\operatorname{Lip}_{\alpha}(X)$, $\operatorname{lip}_{\alpha}(X)$ be defined as in Example 3, but now with the $\Sigma$-norm; this is with the norm defined by

$$
\|f\|=\|f\|_{\infty}+\|f\|_{d^{\alpha}} .
$$

Rao and Roy $[20]$ proved that any isometry from the complex $\operatorname{Lip}_{1}[0,1]$ onto itself is canonical and asked whether the same holds in general. To answer this question let $X, Y$ be compact metric spaces, let $A$ be equal to a complex $\operatorname{Lip}_{\alpha}(X)$ or $\operatorname{lip}_{\alpha}(X)$ space and let $B$ be equal to a complex $\operatorname{Lip}_{\alpha^{\prime}}(Y)$ or $\operatorname{lip}_{\alpha^{\prime}}(Y)$ space. We prove that any isometry $\Phi$ from $A$ onto $B$ is canonical and hence of the form

$$
\Phi f(y)=c f \circ \varphi(y), \quad f \in A, y \in Y,
$$

where $|c|=1$ and $\varphi$ is an isometry from $Y$ onto $X$. To this end, by Theorem 4, it is sufficient to prove that for any such isometry $\Phi, \Phi(\mathbf{1})=\chi$ is a constant function of norm one.

Put

$$
y=\beta(Y \times Y-\{(y, y): y \in Y\}),
$$

define $T: B \rightarrow C(y)$ by

$$
T g\left(y_{1}, y_{2}\right)=\frac{g\left(y_{1}\right)-g\left(y_{2}\right)}{d^{\alpha^{\prime}}\left(y_{1}, y_{2}\right)}
$$

and for any $g \in B$ let us denote by $\tilde{g}$ an element of $C(Y \times \mathcal{Y} \times S)$ defined by

$$
\tilde{g}(y, \omega, \beta)=g(y)+\beta T g(\omega) .
$$

As we noticed in the introduction the map $g \mapsto \tilde{g}$ is an isometric embedding from $B$ onto $\tilde{B} \subset C(Y \times Y \times S)$ and any element $G$ of ext $B^{*}$ is of the form

$$
G(g)=\gamma \tilde{g}(y, \omega, \beta)=\gamma g(y)+\gamma \beta T g(\omega) .
$$


Fix $\left(y_{0}, \omega_{0}, \beta_{0}\right) \in Y \times \omega \times S$ and let $\mu$ be any norm one measure on $Y \times \mathcal{Y} \times S$ which represents, on $\tilde{B}$, evaluation at the point $\left(y_{0}, y_{0}, \beta_{0}\right)$. Considering any function $g_{0} \in B$ which peaks exactly at the point $y_{0}$ it is easy to notice that $\mu$ is concentrated on the set $\left\{y_{0}\right\} \times y \times S$ and that for any $0 \leq \vartheta<2 \pi$ a measure $\mu_{\vartheta}$ defined on $Y \times \mathcal{Y} \times S$ by

$$
\mu_{\vartheta}(E)=\mu\left(\left\{(y, \omega, \beta):\left(y, \omega, e^{i \vartheta \beta}\right) \in E\right\}\right)
$$

represents, on $\tilde{B}$, the functional of the evaluation at the point $\left(y_{0}, \omega_{0}, e^{-i \vartheta \beta_{0}}\right)$. Hence we can define a map $\Psi_{\vartheta}: \operatorname{ext} B^{*} \rightarrow \operatorname{ext} B^{*}$ by

$$
\Psi\left(\gamma_{(y, \omega, \beta)}^{\delta}\right)=\gamma^{\delta}\left(y, \omega, e^{i \vartheta \beta}\right)
$$

For any $F \in \operatorname{ext} A^{*}$ we have $|F(1)|=1$, hence for any $G \in \operatorname{ext} B^{*}$ we also have $|G(\chi)|=1$. Let $G=\delta_{y}+\beta \delta_{\omega} \circ T \in \operatorname{ext} B^{*}$. For any $0 \leq \vartheta<2 \pi$ we have

$$
1=\left|\Psi_{\vartheta}(G)(\chi)\right|=\left|\chi(y)+e^{i \vartheta} \beta T(\chi)(\omega)\right|,
$$

hence for any such $G$ we have two possibilities:

$$
\chi(y)=0 \text { and }|T(\chi)(\omega)|=1
$$

or

$$
|\chi(y)|=1 \text { and } T(\chi)(\omega)=0 .
$$

Since $1=\|\chi\|=\|\chi\|_{\infty}+\|T(\chi)\|_{\infty}$ we get that the second possibility always holds, this means that $T(\chi) \equiv 0$ and we are done.

5. Beside the extensions of the surjective Banach-Stone theorem for various function spaces many authors also considered injective isometries between classical Banach spaces (see for example $[\mathbf{2}, \mathbf{9}, \mathbf{1 0}, \mathbf{1 1}, \mathbf{1 3}]$ ) and isomorphisms with a small bound (see $[1,2,4,6,7,11,13,19]$ ). These problems in general seem to be much harder and the authors do not know whether the similar general schemes can be produced. The following example shows however that even for a very simple function space an injective isometry may be "very uncanonical." Let $A$ be equal to $C^{1}[0,1]$ with the $M$-norm. Let $\varphi$ be a continuous map from $[0,1]$ onto $A_{1}^{*}$ equipped with the weak-* topology. We define $\Phi: A \rightarrow A$ by

$$
\Phi(f)(t)=\int_{0}^{t} \varphi(x)(f) d x .
$$

On the other hand, if we assume that the injective isometry from the above space $A$ into itself preserves the constant function then it is automatically surjective and of the canonical form. This can be proven exactly by the same arguments as used in $[\mathbf{1 2}]$.

\section{REFERENCES}

1. D. Amir, On isomorphisms of continuous function spaces, Israel J. Math. 3 (1965), 205-210.

2. Y. Benyamini, Small into-isomorphisms between spaces of continuous functions, Proc. Amer. Math. Soc. 83 (1981), 479-485.

3. M. Cambern, Isometries of certain Banach algebras, Studia Math. 25 (1965), 217-225.

4. __ On isomorphisms with a small bound, Proc. Amer. Math. Sor. 18 (1967), 1062-1066.

5. M. Cambern and V. D. Pathak, Isometries of spaces of differentiable functions, Math. Japon. 26 (1981), 253-260. 
6. _ Isomorphisms of spaces of differentiable functions, Rev. Roumaine Math. Pures Appl. 27 (1982), 737-743.

7. B. Cengiz, A generalization of the Banach-Stone theorem, Proc. Amer. Math. Soc. 40 (1973), 426-430.

8. N. Dunford and J. T. Schwartz, Linear operators, Interscience, New York, 1958.

9. M. el-Gebeily and J. Wolfe, Isometries of the disc algebra, Proc. Amer. Math. Soc. 93 (1985), 697-702.

10. W. Holsztyński, Continuous mapping induced by isometries of spaces of continuous functions, Studia Math. 26 (1966), 133-136.

11. K. Jarosz, Into isomorphisms of spaces of continuous functions, Proc. Amer. Math. Soc. 94 (1985), 65-71.

12. __ Isometries in semisimple, commutative Banach algebras, Proc. Amer. Math. Soc. 90 (1985), 65-71.

13. __ Perturbations of Banach algebras, Lecture Notes in Math., vol. 1120, Springer-Verlag, 1985.

14. $\ldots, H^{\infty}(D)$ is stable, Bull. London Math. Soc. (to appear).

15. K. de Leeuw, Banach spaces of Lipschitz functions, Studia Math. 21 (1964), 55-66.

16. M. Nagasawa, Isomorphisms between commutative Banach algebras with application to rings of analytic functions, Kōdai Math. Sem. Rep. 11 (1959), 182-188.

17. V. D. Pathak, Isometries of $C^{n}[0,1]$, Pacific J. Math. 94 (1981), 211-222.

18. __ Linear isometries of spaces of absolutely continuous functions, Canad. J. Math. 34 (1982), 298-306.

19. V. D. Pathak and M. H. Vasavada, Isometries and isomorphisms of Banach spaces of differentiable functions (submitted).

20. N. V. Rao and A. K. Roy, Linear isometries of some function spaces, Pacific J. Math. 38 (1971), 177-192.

21. A. K. Roy, Extreme points and linear isometries of the Banach spaces of Lipschitz functions, Canad. J. Math. 20 (1968), 1150-1164.

22. M. H. Vasavada, Closed ideals and linear isometries of certain function spaces, Ph.D. thesis, Univ. of Wisconsin, 1969.

Institute of Mathematics, Warsaw University, P.K. I N. 9P, 00-901, WaRsaw, POLAND (Current address of V. D. Pathak)

Department of Applied Mathematics, Faculty of Technology and EngiNEERING, M. S. UNIVERSITY OF BARODA, BARODA 3900 001, INDIA

Current address (K. Jarosz): Department of Mathematics, University of California, Santa Barbara, California 93106 